\title{
https://doi.org/10.46813/2020-130-050 \\ BRAGINSKII EQUATIONS FOR HOT RELATIVISTIC PLASMAS: MIXED APPROACH
}

\author{
I. Marushchenko, N.A. Azarenkov \\ V.N. Karazin Kharkiv National University, Kharkiv, Ukraine
}

In the paper, the Braginskii equations for relativistic electrons in hot plasmas with slow macroscopic fluxes are derived. This consideration is suitable for description of the typical fusion plasma with the temperatures of about several tens of kiloelectronvolt, when the plasma rotation and the longitudinal currents should be taken into account. Contrary to other papers devoted to classical description of transport processes in fusion devices, as well as to fully relativistic description of the astrophysical objects, we propose the mixed approach with fully relativistic kinetics for the hot electrons and non-relativistic macroscopic fluxes. The obtained form of the Braginskii equations includes all important features of relativistic hydrodynamics, has the same form as the classical representation, which is currently implemented into modern transport codes, and can easily replace the latter.

PACS: 52.55.Dy, 52.25.Fi, 52.27.Ny

\section{INTRODUCTION}

Due to the progress in fusion research in last decades [1-4], the typical temperature of amagnetically confined plasma in large tokamaks and stellarators can already reach the range of $10 \ldots 20 \mathrm{keV}$. In next-generation fusion projects, such as the ITER [5] and DEMO [6] tokamaks the temperatures should be even higher, up to $60 \mathrm{keV}$, depending on the scenario.

Formally, each scenario mentioned above satisfies the condition $T_{e} \ll m_{e} c^{2}$. At the same time, there are many effects and processes in which relativistic effects make a non-negligible contribution and must be taken into account. For example, physics of electron cyclotron heating and current drive [7] and radiation losses in hot plasmas [8].

In addition, in the "inertial fusion" [9], when a plasma is created by a short laser pulse of extremely high power, the energies of electrons in exploding plasma streams can reach hundreds of $\mathrm{keV}$, which is already quite relativistic.

It is also important to mention the projects of a neutronic fusion [10-12], based on the reactions $\mathrm{D}-\mathrm{He}^{3}$ and $p-B^{11}$, in which temperatures of the order of $50 \ldots 70 \mathrm{keV}$ (and even higher)should be maintained, which requires a fully relativistic description.

In general, the basic theory for describing relativistic plasmas is well developed, see [13, 14]. A number of theoretical studies of relativistic kinetic and transport have been carried out. Usually, attention is focused on astrophysical objects [15], where a fully relativistic description is required and it is common to apply the covariant formalism with the 4-vectors in the Minkowski space [14]. In particular, in the literature one can find papers devoted to the description of fully relativistic and ultra-relativistic plasmas and plasma with flows; see, for example, [16]. In addition, fully relativistic hydrodynamic has already been developed [17]. At the same time, the plasma generated in laboratory, for example in fusion devices, does not require such a formalism and can be well described without refence to the Lorentz invariance [18-21]. The present paper deals with the case of quite practical needs and the results obtained here can be easily implemented in any transport code.

Below, we consider a quite typical situation in fusion plasmas where the macroscopic flows exist. These flows can be associated with plasmarotation or longitudinal electriccurrent in the magnetized plasmas. The main feature of this kind of flows is that $V \ll v_{t e}$ and $v_{t e}<c$. In other words, hot plasma electrons can be relativistic, while the flows are characterized by the classical velocities.

There are two ways to find the optimal model for describing transport processes in such plasmas: i) start with a fully relativistic model obtained with Lorentz invariance (see, for example, [16]) and reduce it to the required level; and ii) derive it directly from the relativistic kinetic equations using Braginskii's method and only those effects that need to be considered. We have found the second method to be simpler and more convenient.

The resulting equations like any other chain of equations for the moments [22, 23] must be closed. This means that in orderto solve the resulting system of equations, it is first necessary to calculate the moments in the rest frame. This can be done by solving the linearized kinetic equation with respect to $\delta f_{e}$. However, this task is not considered here.

Below, we derive the Braginskii equations for hot plasmas with relativistic electrons and non-relativistic macroscopic fluxes.

\section{DRIFTING JÜTTNER-MAXWELLIAN AND QUASI-RELATIVISTIC GALILEAN TRANSFORMATION}

We start from the standard relativistic kinetic equation for electrons in plasmas which can be written as following [14],

$\frac{\partial f_{e}}{\partial t}+\frac{\mathbf{u}}{\gamma} \cdot \nabla f_{e}+\frac{e}{m_{e}}\left(\mathbf{E}+\frac{\mathbf{u}}{c \gamma} \times \mathbf{B}\right) \cdot \frac{\partial f_{e}}{\partial \mathbf{u}}=C_{e}\left(f_{e}\right)$,

where $f_{e}$ - the electron distribution function,

$\boldsymbol{u}=\mathbf{v} \gamma-$ the moment of electron per unit mass and 
$\gamma=\sqrt{1+u^{2} / c^{2}}, \mathbf{E}$ and $\mathbf{B}-$ the electric and magnetic fields, respectively, $\quad C_{e}\left(f_{e}\right) \equiv C_{e e}\left(f_{e}\right)+C_{e i}\left(f_{e}\right)-$ relativistic Coulomb collisional operator which describes the collisions of electrons with themselves and with the ions. Generally, in Eq. (1) also the external sources of particles and energy, $S_{\text {ext }}$, has to be accounted. However, since it seems rather a trivial point, we omit this term here and mention it below only if necessary.

Formally, in order to derive the equations for the set of moments of the distribution function, we do not have to solve the Eq. (1). However, this solution is required in order to close the system of transport equations. Since the closure is not the point of this paper, we only briefly discuss the features of $f_{e}$.

The object of our investigation are quite slow processes with characteristic times sufficient for relaxation of plasmas to the thermodynamic equilibrium. As consequence, we can suppose that the ions are well described by the (classical) Maxwellian, while the electrons may have only small deviations from the equilibria. As usually, all these deviations are induced by the thermodynamic forces (plasma parameters gradients) and the external forces. As consequence, the electron distribution function can be represented in standard form, $f_{\mathrm{e}}=f_{\mathrm{e} 0}+\delta f_{\mathrm{e}}$, where $\delta f_{\mathrm{e}}$ - small deviation from the drifting Jüttner-Maxwellian, $f_{e 0}=C_{e J M} \frac{n_{e}}{\pi^{3 / 2} u_{t e}^{3}} \exp \left[-\mu \gamma_{0}\left(\gamma-\frac{1}{\gamma_{0}}-\frac{V_{\alpha} u_{\alpha}}{c^{2}}\right)\right]$,

where $\boldsymbol{V}$ - the macroscopic flux velocity of electrons and $\gamma_{0}=\left(1-V^{2} / c^{2}\right)^{-1 / 2}$ - the respective relativistic factor, $u_{t e}=p_{t e} / m_{e}-$ the thermal moment per unit mass with $p_{t e}=\left(2 m_{e} T_{e}\right)^{1 / 2}\left(u_{t e}\right.$ seems equal to the thermal velocity, but it is notlimited by the speed of light), and $\mu_{r}=m_{e} c^{2} / T_{e}$. Here and below, we apply the rule of summation over the repetitive indexes. The normalizing factor is, respectively

$$
C_{e J}=\sqrt{\frac{\pi}{2 \mu_{r}}} \frac{e^{-\mu_{r}}}{K_{2}\left(\mu_{r}\right)}=1-\frac{15}{8 \mu_{r}}+\mathcal{O}\left(\mu_{r}^{-2}\right) .
$$

where $K_{2}(x)$ is the modified Bessel function of second kind.

The form of representation of $f_{e 0}$ in Eq. (2) is chosen in such a way that a recovering of the classical (drifting) Maxwellian in the case of both $T_{e} / m_{e} c^{2} \rightarrow 0$ and $V^{2} / c^{2} \rightarrow 0$ would be most evident.

The relation between $f_{e 0}$ and $\delta f_{\mathrm{e}}$ is chosen in such a way that $\delta f_{\mathrm{e}}$ does not contribute to the integrals for density

$$
n_{e}=\int f_{e} \mathrm{~d}^{3} u=\int f_{e 0} \mathrm{~d}^{3} u,
$$

as well as for the macroscopic flux

$$
\boldsymbol{\Gamma}_{e}=n_{e} \mathbf{V}=\int \mathbf{v} f_{e} \mathrm{~d}^{3} u=\int \mathbf{v} f_{e 0} \mathrm{~d}^{3} u .
$$

Following the Braginskii method [22, 23], let us account a presence of the macroscopic flux with the help of transformation to the frame of reference which moves with the flux velocity $\mathbf{V}$. Since we are interested in the slow non-relativistic fluxes, it seems natural to apply the classical Galilean velocity transformation, $v_{\alpha}=v_{\alpha}^{\prime}+V_{\alpha}$. However, the relativistic nature of the moving object (i.e. the hot electrons themselves) means that the only right way is the reduction of the general Lorentz transformation (see, for example, [24]), taking $\gamma_{0} \simeq 1+V^{2} / 2 c^{2}$ and keeping only the lowest order of $V$,

$$
\begin{aligned}
u_{\alpha} & =u_{\alpha}^{\prime}+V_{\alpha} \gamma^{\prime}, \\
\gamma & =\gamma^{\prime}+\frac{V_{\alpha} u_{\alpha}^{\prime}}{c^{2}}+\frac{V^{2}}{2 c^{2}} .
\end{aligned}
$$

Since this approach has a lower order with respect to $V$ than the weakly relativistic approach [24], we call this transformation a quasi-relativistic Galilean.

Similarly, the drifting Jüttner-Maxwell distribution (DJM) also can be reduced,

$$
f_{e 0}=C_{e J M} \frac{n_{e}}{\pi^{3 / 2} u_{t e}^{3}} \exp \left[-\mu\left(\gamma-1-\frac{V_{\alpha} u_{\alpha}}{c^{2}}\right)-\frac{m_{e} V^{2}}{2 T_{e}}\right] .
$$

This approach includes all required effects. Below we will use this form of reduced DJM.

For clarity, however, one specific point has to be emphasized. While the precise Lorentz transformation when applied to the standard DJM precisely cancel the drift in the moving coordinate system (exactly the same as in the classical case with the classical Galilean transformation), application of the quasi-relativistic Galilean transformation, Eq. (6), to the reduced DJM, given by Eq.(7), leads to only approximate canceling of thedrift. However, it can be easily shown that the residual term in exponentis of order $\left(m_{e} V^{2} / T_{e}\right)\left(u^{\prime 2} /\right.$ $c^{2}$ ), which is very small since both conditions $m_{e} V^{2} /$ $T_{e} \ll 1$ and $u^{\prime 2} / c^{2} \ll 1$ are well satisfied by definition. Actually, this result confirms a validity of the proposed approach.

For calculation of the moments in the next chapter, the Jacobian of the coordinate transformation is required. It can be found from the invariance of the phase-space volume and can be written as following:

$$
\frac{d^{3} u}{\gamma}=\frac{d^{3} u^{\prime}}{\gamma^{\prime}} \text { and } \frac{\gamma}{\gamma^{\prime}} \simeq 1+\frac{V_{\alpha} v_{\alpha}^{\prime}}{c^{2}}+\frac{V^{2}}{2 c^{2}} .
$$

\section{THE EQUATIONS FOR MOMENTS}

In this chapter, the moments of Eq. (1) are calculated. For compactness, we introduce the following notation

$$
\langle A\rangle=\frac{1}{n_{e}} \int A f_{e} \mathrm{~d}^{3} u \text { and }\left\langle A^{\prime}\right\rangle=\frac{1}{n_{e}} \int A^{\prime} f_{e} \mathrm{~d}^{3} u^{\prime} .
$$

One can checkthat $\left\langle v_{\alpha}\right\rangle=V_{\alpha}$ and $\left\langle v_{\alpha}^{\prime}\right\rangle \simeq 0$.

Direct integration of Eq. (1) leads to a continuity equation, which describes a conservation of the number of particles. In terms of the first moments, Eqs. (4, 5), this equation can be written as

$$
\frac{\partial n_{e}}{\partial t}+\operatorname{div} \boldsymbol{\Gamma}_{e}=\left(\dot{n}_{e}\right)_{\mathrm{ext}}
$$

and there is no difference from the standard form. Here, in the right-hand-side, a possible presence of the external source is accounted.

In order to calculate the next moments, such as the momentum, $\left\langle m_{e} u_{\alpha}\right\rangle$, kinetic energy, $\left\langle m_{e} c^{2}(\gamma-1)\right\rangle$, and the viscosity tensor, $\left\langle m_{e} u_{\alpha} v_{\beta}\right\rangle$, with the drift velocity, one has to define them in the rest frame, i.e. 
the frame with no drift. The density of averaged kinetic energy of relativistic electrons can be written as [19]

$$
W_{e}=n_{e} m_{e} c^{2}\left\langle\gamma^{\prime}-1\right\rangle=\left(\frac{3}{2}+\mathcal{R}\right) p_{e},
$$

where $p_{e}=\frac{1}{3} n_{e} m_{e}\left\langle\frac{u^{\prime 2}}{\gamma^{\prime}}\right\rangle=n_{e} T_{e}$ is the pressure and

$\mathcal{R}\left(\mu_{r}\right)=\mu_{r}\left(\frac{K_{3}}{K_{2}}-1\right)-\frac{5}{2} \simeq \frac{15}{8 \mu_{r}}+\mathcal{O}\left(\mu_{r}^{-2}\right)$

is the additional relativistic correction to the classical definition of energy density, $W_{e}=\frac{3}{2} n_{e} T_{e}$.

We introduce also the heat flux:

$$
q_{\alpha}=n_{e}\left\langle v_{\alpha}^{\prime} m_{e} c^{2}\left(\gamma^{\prime}-1\right)\right\rangle
$$

Now, it is easy to calculate the next moments, related to the rest frame,

$$
\begin{aligned}
n_{e} m_{e}\left\langle u_{\alpha}^{\prime}\right\rangle & =\frac{q_{\alpha}}{c^{2}} \\
n_{e} m_{e}\left\langle\frac{u_{\alpha}^{\prime} u_{\beta}^{\prime}}{\gamma^{\prime}}\right\rangle & =\pi_{\alpha \beta}+\delta_{\alpha \beta} p_{e} .
\end{aligned}
$$

One can note that first moment in Eq. (14) is non-zero only due to the relativistic effects, while in the classical limit $\left\langle u_{\alpha}^{\prime}\right\rangle \rightarrow\left\langle v_{\alpha}^{\prime}\right\rangle=0$. Second moment in Eq. (14) corresponds to the viscosity tensor, non-diagonal part of which can be written similar to the classical definition $[22,23]$,

$$
\pi_{\alpha \beta}=n_{e} m_{e}\left(\left\langle\frac{u_{\alpha}^{\prime} u_{\beta}^{\prime}}{\gamma^{\prime}}\right\rangle-\frac{1}{3}\left\langle\frac{u^{\prime 2}}{\gamma^{\prime}}\right\rangle \delta_{\alpha \beta}\right) .
$$

Apart from that, we need to consider the terms with the collision operator. Since we take into account only the Coulomb collisions of electrons with electrons and ions, the number of particles is conserved automatically. Moreover, collisions of electrons with themselves conserve both the momentum and energy. As consequence, in the following only the electrons-ions collisional operator, $C_{e i}\left(f_{e}\right)$, is required.

Again, firstly we consider only the rest frame. For that, we introduce the collisional friction force between the electrons and ions,

$$
R_{\alpha}^{e i}=\int m_{e} u_{\alpha}^{\prime} C_{e i}\left(f_{e}\right) \mathrm{d}^{3} u^{\prime},
$$

the stress tensor generated by the electron-ion collisions,

$$
F_{\alpha \beta}^{e i}=\int m_{e} v_{\alpha}^{\prime} u_{\beta}^{\prime} C_{e i}\left(f_{e}\right) \mathrm{d}^{3} u^{\prime}
$$

and the collisional rate of heat flux generated,

$$
G_{\alpha}^{e i}=\int v_{\alpha}^{\prime} m_{e} c^{2}\left(\gamma^{\prime}-1\right) C_{e i}\left(f_{e}\right) \mathrm{d}^{3} u^{\prime} .
$$

Additionally, we introduce the rate of energy exchange between the relativistic electrons and classical ions. This process is significant only if $T_{e}$ and $T_{i}$ are different. In this case, the contribution from $\delta f_{e}$ is negligible, and the energy exchange can be explicitly calculated with the relativistic Jüttner-Maxwellian distribution of electrons and the classical Maxwellian distribution of ions [18],

$$
\begin{aligned}
P^{e i} & =\int m_{e} c^{2}\left(\gamma^{\prime}-1\right) C_{e i}\left(f_{e 0}\right) \mathrm{d}^{3} u^{\prime} \\
& =P_{(c l)}^{e i} C_{e J M}\left(\mu_{r}\right)\left(1+\frac{2}{\mu_{r}}+\frac{2}{\mu_{r}^{2}}\right),
\end{aligned}
$$

(in somewhat different form it was obtained also in [17]).
Here, $P_{(c l)}^{e i}$ is the classical value [23],

$P_{(c l)}^{e i}=-\frac{4}{\sqrt{\pi}} \nu_{e 0} \frac{m_{e}}{m_{i}} n_{i} Z_{i}^{2}\left(T_{e}-T_{i}\right) \propto-\frac{T_{e}-T_{i}}{T_{e}^{3 / 2}}$,

with $v_{e 0}$ as the standard collision frequency (not shown here).

\section{THE BALANCE EQUATIONS}

Since the final aim of this work is to obtain the equations which describe the force and energy balance in the laboratory frame of reference (equation of the particles balance is trivial and is given already; see Eq. (10)), we consider the moments that appear during an integration of the kinetic equation Eq. (1) with the weights $m_{e} u_{\alpha}$ and $m_{e} c^{2}(\gamma-1)$. Apart from that, also the moments $\left\langle m_{e} u_{\alpha} v_{\beta}\right\rangle$ and $\left\langle v_{\alpha} m_{e} c^{2}(\gamma-1)\right\rangle$ arise.

Using the Eqs. (6) and (8), we calculate the first moment, i.e. the averaged momentum. Here and below, the terms of order $V^{2} / c^{2}$ are neglected in final expressions and only the lowest order of $V$ is taken into account:

$$
\begin{aligned}
& n_{e} m_{e}\left\langle u_{\alpha}\right\rangle=n_{e} m_{e}\left\langle\left(1+\frac{V_{\beta} v_{\beta}^{\prime}}{c^{2}}+\frac{V^{2}}{2 c^{2}}\right)\left(u_{\alpha}^{\prime}+\gamma^{\prime} V_{\alpha}\right)\right\rangle \\
& \simeq n_{e} m_{e} V_{\alpha}+\frac{1}{c^{2}}\left[q_{\alpha}+\left(\frac{5}{2}+\mathcal{R}\right) n_{e} T_{e} V_{\alpha}+\pi_{\alpha \beta} V_{\beta}\right] .
\end{aligned}
$$

For convenience, all relativistic corrections are grouped in brackets. One can see that the thermal force related to $q_{\alpha} / c^{2}$ is not canceled by $V=0$.

As was mentioned above, for the force balance the additional moment is required. Formally, this moment corresponds to the viscosity tensor:

$$
\begin{aligned}
& n_{e} m_{e}\left\langle u_{\alpha} v_{\beta}\right\rangle=n_{e} m_{e}\left\langle\left(u_{\alpha}^{\prime}+\gamma^{\prime} V_{\alpha}\right)\left(v_{\beta}^{\prime}+V_{\beta}\right)\right\rangle \\
& \simeq \Pi_{\alpha \beta}+\frac{1}{c^{2}}\left(q_{\alpha} V_{\beta}+q_{\beta} V_{\alpha}\right) .
\end{aligned}
$$

where, following the classical definition,

$$
\Pi_{\alpha \beta}=n_{e} m_{e} V_{\alpha} V_{\beta}+\pi_{\alpha \beta}+p_{e} \delta_{\alpha \beta} .
$$

Please note that this expression, apart from the term in the square bracket, contains the relativistic correction also in the viscosity tensor $\pi_{\alpha \beta}$; see Eq. (15).

In order to simplify the calculation of the term, related to interaction with the electric and magnetic fields, it is convenient to use the conservative form of Eq. (1),

$$
\int m_{e} u_{\alpha} \frac{\partial}{\partial u_{\beta}}\left(\dot{u}_{\beta} f_{e}\right) \mathrm{d}^{3} u=-e n_{e}\left(E_{\alpha}+\frac{1}{c}[\mathbf{V} \times \mathbf{B}]_{\alpha}\right)
$$

This term is precise in any approach.

The last term in the force balance is the collisional friction. In the lowest order with respect to $V$ the result can be written as

$$
\begin{aligned}
& \int m_{e} u_{\alpha} C_{e i}\left(f_{e}\right) \mathrm{d}^{3} u \\
& =\int m_{e}\left(1+\frac{V_{\beta} v_{\beta}^{\prime}}{c^{2}}+\frac{V^{2}}{2 c^{2}}\right)\left(u_{\alpha}^{\prime}+\gamma^{\prime} V_{\alpha}\right) C_{e i}\left(f_{e}\right) \mathrm{d}^{3} u^{\prime} \\
& \simeq R_{\alpha}^{e i}+\frac{1}{c^{2}}\left(P^{e i} V_{\alpha}+F_{\alpha \beta}^{e i} V_{\beta}\right) .
\end{aligned}
$$

Finally, we obtain the balance of forces, which can also be called the momentum conservation equation: 


$$
\begin{aligned}
& \frac{\partial}{\partial t}\left(n_{e} m_{e} V_{\alpha}+\frac{1}{c^{2}}\left[q_{\alpha}+\left(\frac{5}{2}+\mathcal{R}\right) n_{e} T_{e} V_{\alpha}+\pi_{\alpha \beta} V_{\beta}\right]\right) \\
& +\frac{\partial}{\partial x_{\beta}}\left(\Pi_{\alpha \beta}+\frac{1}{c^{2}}\left(q_{\alpha} V_{\beta}+q_{\beta} V_{\alpha}\right)\right) \\
& =e n_{e}\left(E_{\alpha}+\frac{1}{c}[\mathbf{V} \times \mathbf{B}]_{\alpha}\right)+R_{\alpha}^{e i}+\frac{1}{c^{2}}\left(V_{\alpha} P^{e i}+V_{\beta} F_{\alpha \beta}^{e i}\right) .
\end{aligned}
$$

This equation is written in such a way that all relativistic corrections are emphasized. The non-relativistic limit can be easily recovered when $c \rightarrow \infty$.

As the next step, let us consider the moments required for the energy balance equation. Again, we calculate the respective moments in the laboratory frame. Let us start from the density of energy:

$$
\begin{aligned}
& n_{e} m_{e} c^{2}\langle(\gamma-1)\rangle \\
& =n_{e} m_{e} c^{2}\left\langle\left(1+\frac{V_{\alpha} v_{\alpha}^{\prime}}{c^{2}}+\frac{V^{2}}{2 c^{2}}\right)\left(\gamma^{\prime}-1+\frac{V_{\beta} u_{\beta}^{\prime}}{c^{2}}\right)\right\rangle \\
& \simeq W_{e}+n_{e} \frac{m_{e} V^{2}}{2}+\frac{3}{c^{2}} q_{\alpha} V_{\alpha} .
\end{aligned}
$$

One can find that with $c \rightarrow \infty$ this expression can be reduced to the classical limit, $\frac{3}{2} n_{e} T_{e}+n_{e} \frac{m_{e} v^{2}}{2}$.

The energy flow can be calculated using the previous results with the same accuracy,

$n_{e} m_{e} c^{2}\left\langle v_{\alpha}(\gamma-1)\right\rangle \simeq q_{\alpha}+\left(\frac{5}{2}+\mathcal{R}\right) n_{e} T_{e} V_{\alpha}+\pi_{\alpha \beta} V_{\beta}$.

Next, for calculation of the term with electric and magnetic field, it is convenient to use the conservative form of kinetic equation. Since the magnetic field does not perform any work, only the electric field contribute to the respective moment,

$$
\int m_{e} c^{2}(\gamma-1) \frac{\partial}{\partial u_{\beta}}\left(\dot{u}_{\beta} f_{e}\right) \mathrm{d}^{3} u=-e n_{e} E_{\alpha} V_{\alpha} .
$$

This term is also precise in any approach.

The rate of collisional change of the energy can be written as

$$
\begin{aligned}
& \int m_{e} c^{2}(\gamma-1) C_{e i}\left(f_{e}\right) \mathrm{d}^{3} u=\int m_{e} c^{2}\left(1+\frac{V_{\alpha} v_{\alpha}^{\prime}}{c^{2}}+\frac{V^{2}}{2 c^{2}}\right) \\
& \times\left(\gamma^{\prime}-1+\frac{V_{\beta} u_{\beta}^{\prime}}{c^{2}}\right) C_{e i}\left(f_{e}\right) \mathrm{d}^{3} u^{\prime} \\
& \simeq P^{e i}+\left(R_{\alpha}^{e i}+\frac{1}{c^{2}} G_{\alpha}^{e i}\right) V_{\alpha} .
\end{aligned}
$$

Finally, combining all terms together, the energy balance equation can be written as following:

$$
\begin{aligned}
& \frac{\partial}{\partial t}\left(W_{e}+n_{e} \frac{m_{e} V^{2}}{2}+\frac{3}{c^{2}} q_{\alpha} V_{\alpha}\right) \\
& +\frac{\partial}{\partial x_{\alpha}}\left[\left(\frac{5}{2}+\mathcal{R}\right) n_{e} T_{e} V_{\alpha}+q_{\alpha}+\pi_{\alpha \beta} V_{\beta}\right] \\
& =e n_{e} E_{\alpha} V_{\alpha}+P^{e i}+\left(R_{\alpha}^{e i}+\frac{1}{c^{2}} G_{\alpha}^{e i}\right) V_{\alpha} .
\end{aligned}
$$

One can see that the obtained set of "fluid" equations, Eqs. (10), (26), and (31), recovers the well-known classical limit. Indeed, in the limit $c \rightarrow \infty$ and $T_{e} /$ $m_{e} c^{2} \rightarrow \infty$, the terms proportional to $1 / c^{2}$ disappear, $\mathcal{R} \rightarrow 0$, and $u \rightarrow v$. Evidently, the equations will be exactly the same as given in $[22,23]$.

\section{CONCLUSIONS}

In the paper, the "fluid" equations for the relativistic electrons in hot plasmas have been derived. We have considered the case when slow flows with characteristic velocities $V \ll v_{t e}$ can be generated in plasma, while the thermal electron velocity is arbitrary and can even reach the speed of light. This condition makes it possible to apply a significantly reduced model, with a fully relativistic description of plasmas electrons and a "classical" description of macroscopic flows.

In order to derive the equations, the method of Braginskii has been applied, with the only difference from the original derivation is that the macroscopic flow was taken into account using the so called quasirelativistic Galilean transformation with an accuracy to the lowest order of flow velocity. The equations are written in such a way that the relativistic contributions manifest itself as additional terms - relativistic corrections.

We have found that the "mixed" approach, i.e. the description of plasma electrons at a fully relativistic level combined with a non-relativistic description of the flows, is completely sufficient for describing this kind of plasmas. Although there is practically no restriction on the electron temperature, the characteristic velocity of a flow is limited by the conditions $V^{2} / c^{2} \ll 1$ and $V \ll v_{t e}$. However, both of these conditions are typically well satisfied for any fusion device by a wide margin.

\section{REFERENCES}

1. International Fusion Research Council (IFRC). Status Report on Fusion Research // Nucl. Fusion. 2005, v. 45 , p. A1.

2. R.J. Hawryluk. Results from deuterium-tritium tokamak confinement experiments // Rev. Mod. Phys. 1998, v. 70, p. 537.

3. A. Dinklage, C.D. Beidler, P. Helander, et al. Magnetic configuration effects on the Wendelstein 7-X stellarator // Nature Phys. 2018, v. 14, p. 855-860.

4. N.B. Marushchenko et al. Main results ofthe first experimental campaign in the W7-X stellarator // Problems of Atomic Sci. and Technol. Series «Plasma Physics»(106). 2016, № 6, p. 3.

5. C. Gormezano et al. ITER, Ch.6: Steady State operation // Nucl. Fusion. 2007, v. 47, p. S285.

6. D.J. Ward. The physics of DEMO // Plasma Physics Control. Fusion. 2010, v. 52, p. 124033.

7. E. Poli, G. Tardini, H. Zohm, et al. Electroncyclotron-current-drive efficiency in DEMO plasmas // Nucl. Fusion. 2013, v. 53, p. 013011.

8. Wen-Jia Chen et al. Relativistic Electron-Electron Bremsstralung in Fusion Plasmas // J. Phys. Soc. Japan. 1982, v. 51, p. 1620.

9. Ph. Nikolai et al. Effect of the plasma-generated magnetic field on relativistic electron transport //Phys. Rev. E. 2011, v. 84, p. 016402.

10. W.M. Nevins. A Review of Confinement Requirements for Advanced Fuels // J. of Fusion Energy. 1998, v. 17, p. 25.

11. P.E. Stott. The feasibility of using D- ${ }^{3} \mathrm{He}$ and D-D fusion fuels // Plasma Phys. Control. Fusion. 2005, v. 47 , p. 1305. 
12. S.V. Putvinski, D.D. Ryutov, P.N. Yushmanov. Fusion reactivity of the $\mathrm{p}-\mathrm{B}^{11}$ plasma revisited // Nucl. Fusion. 2019, v. 59, p. 076018.

13. S.T. Beliaev, G.I. Budker. Relativistic kinetic equation // Dokl. Akad. Nauk USSR. 1956, v. 6, p. 807810. English transl.: Soviet Phys. Doklady. 1956, v. 1, p. 218.

14. S.R. de Groot, W.A. van Leewen, Ch.G. van Weert. Relativistic Kinetic Theory. North-Holland Publishing Company, Amsterdam, 1980.

15. Y.B. Zeldovich, I. Novikov. Relativistic Astrophysics University of Chicago Press, Chicago, 1983.

16. D.I. Dzhavakhishvili, N.L. Tsintsadze. Transport phenomena in a completely ionized ultra relativistic plasma // Sov. Phys. JETP. 1973, v. 37, p. 666.

17. J.M. Ten Barge, R.D. Hazeltine, S.M. Mahajan. Fluid model for relativistic, magnetized plasmas // Phys. Plasmas. 2008, v. 15, p. 062112.

18. I. Marushchenko, N.A. Azarenkov, N.B. Marushchenko. On stability of collisional coupling between relativistic electrons and ions in hot plasmas // Phys. Plasmas. 2012, v. 19, p. 112109.
19. I. Marushchenko, N.A. Azarenkov, N.B. Marushchenko. Relativistic neoclassical radial fluxes in the $1 / \mathrm{v}$ regime // Plasma Phys. Control. Fusion. 2013, v. 55, p. 085005 .

20. I. Marushchenko, N.A. Azarenkov, N.B. Marushchenko. Relativistic neoclassical fluxes in hot plasmas // Problems of Atomic Science and Technology. Series «Plasma Physics» (83). 2013, № 1, p. 67.

21. I. Marushchenko, N.A. Azarenkov. Relativistic mono-energetic transport coefficients in hot plasmas // Problems of Atomic Science and Technology. Series «Plasma Physics» (86). 2013, № 4, p. 112.

22. S.I. Braginskii. Review of Plasma Physics / Edited by M.A. Leontovich, Consultant Bureau, New York, 1965, v. 1, p. 205.

23. P. Helander, D.J. Sigmar. Collisional Transport in Magnetized Plasmas Cambridge. University Press, 2002.

24. L.D. Landau, E.M. Livshitz. The Classical Theory of Fields. v. 2 Butterworth-Heinemann, 4th Edition, 1987.

Article received 15.10.2020

\title{
УРАВНЕНИЯ БРАГИНСКОГО ДЛЯ ГОРЯЧЕЙ РЕЛЯТИВИСТСКОЙ ПЛАЗМЫ С МЕДЛЕННЫМИ ПОТОКАМИ: СМЕШАННОЕ ПРИБЛИЖЕНИЕ
}

\author{
И. Марущенко, Н.А. Азаренков
}

Выводятся уравнения Брагинского для релятивистских электронов в горячей плазме с медленными макроскопическими потоками. Это рассмотрение подходит для описания типичной термоядерной плазмы с температурами в десятки килоэлектронвольт, когда необходимо учитывать вращение плазмы и продольные потоки. В отличие от других работ, посвященных классическому описанию процессов переноса в термоядерных устройствах, а также полностью релятивистскому описанию астрофизических объектов, мы предлагаем смешанный подход с полностью релятивистской кинетикой для горячих электронов и нерелятивистскими макроскопическими потоками. Полученная форма уравнений Брагинского включает в себя все важные особенности релятивистской гидродинамики, имеет тот же вид, что и классическое представление, которое в настоящее время реализовано в современных транспортных кодах, и может легко заменить последнее.

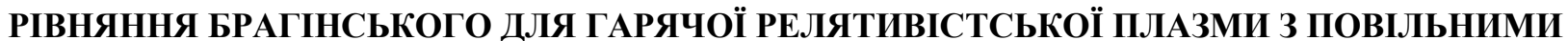 ПОТОКАМИ: ЗМШШАНЕ НАБЛИЖЕННЯ}

\section{I. Марущенко, М.О. Азарєнков}

Виведені рівняння Брагінського для релятивістських електронів у гарячій плазмі 3 повільними макроскопічними потоками. Запропонований розгляд підходить для опису лабораторної термоядерної плазми 3 температурами в десятки кілоелектронвольт, коли слід враховувати обертання плазми та поздовжні потоки. На відміну від інших робіт, присвячених класичному опису транспортних процесів у термоядерних пристроях, а також повному релятивістському опису астрофізичних об'єктів, ми пропонуємо змішане наближення 3 повністю релятивістською кінетикою для гарячих електронів та нерелятивістськими макроскопічними потоками. Отримана форма рівнянь Брагінського включає всі важливі особливості релятивістської гідродинаміки, має ту ж форму, що і класичне подання, яке в даний час впроваджено в сучасні транспортні коди, і може легко замінити останнє. 